\title{
Turbulent viscosity profile of drag reducing rod-like polymers ${ }^{\star}$
}

\author{
Dilafruz Kulmatova ${ }^{1}$, Ferhat Hadri ${ }^{2}$, Sylvain Guillou ${ }^{3}$, and Daniel Bonn ${ }^{1, a}$ \\ 1 Institute of Physics, University of Amsterdam, Science Park 904, 1098 XH Amsterdam, The Netherlands \\ 2 Laboratoire d'Ingénierie des Systèmes de Versailles (LISV), 10/12 Avenue de l'Europe 78140 Velizy, France \\ 3 LUSAC, University of Caen Normandy, 60 Rue Max Pol Fouchet, CS 20082, 50130 Cherbourg-Octeville, France
}

Received 17 March 2018 and Received in final form 7 May 2018

Published online: 12 December 2018

(c) The Author(s) 2018. This article is published with open access at Springerlink.com

\begin{abstract}
Recent theories of drag reduction in wall turbulence assumed that the presence of the polymer leads to an effective viscosity, which increases linearly with the distance from the wall. Such a linear viscosity profile reduces the Reynolds stress (i.e., the momentum flux to the wall), which leads to drag reduction. For the usual flexible polymers employed in drag reduction, the effective viscosity is however a strongly non-linear effect that is difficult to quantify. We therefore investigate the turbulent drag reduction characteristics of a stiff rod-like polymer for which any effective viscosity changes are only due to the orientation of the polymers. The results show that close to the walls the polymers orient and the viscosity is low, whereas in the bulk the polymers are randomly oriented and the effective viscosity is high. This indeed leads to a reduction of the Reynolds stress and hence to a drag reduction.
\end{abstract}

\section{Introduction}

Polymer drag reduction has found applications in many fields, primarily in pipe-flows, but also in marine and biomedical applications [1]. The drag reduction effect is generally achieved by using a minute amount of polymer additive in a turbulent fluid flow; the addition of the polymer results in a large reduction in the frictional drag in pipes and channels. Drag reduction by polymer additives has been extensively studied because of the significant potential benefits to many systems such as long-distance transportation of liquids, oil-well operations, transportation of suspensions and slurries, district heating and cooling, fire-fighting operations and irrigation. The use of polymer additives to enhance flow in petroleum pipelines has received the greatest attention due to its great commercial success. Despite a large amount of experimental and simulation approaches, the nature of the drag reduction phenomenon is still incompletely understood and remains a matter of debate [2-5]. The classical explanation of drag reduction is based on the notion of an increased effective viscosity. As suggested by Lumley [6,7], stretching randomly coiled polymers increases the effective viscosity in (part of the) boundary layer. This increase in viscosity leads to a thickening of the buffer layer separating the

\footnotetext{
* Contribution to the Topical Issue "Flowing Matter, Problems and Applications", edited by Federico Toschi, Ignacio Pagonabarraga Mora, Nuno Araujo, Marcello Sega.

${ }^{a}$ e-mail: d.bonn@uva.nl
}

viscous layer and therefore leads to a reduction in wall friction. Recently, much progress was by formulating a linear effective viscosity model for drag reduction [8-10]. Both experiments and theory [8-10] agree with the idea that the presence of the polymer leads to an effective viscosity that increases linearly with the distance from the wall. Such a linear viscosity profile then reduces the Reynolds stress in the elastic sublayer, resulting in a reduction of the momentum flux from bulk flow to the wall compared to that for Newtonian which in turn leads to drag reduction. However the origin of the linear profile is less clear. In [4, 8-11], the effective viscosity profile was evaluated from the momentum and energy equations; after a number of approximations the effective viscosity was estimated to be a linear function of the distance from the wall. Such a linear effective viscosity profile should then originates from polymer stretching in the wall-normal direction; this stretching leads to an elevated elongational viscosity that is responsible for the increase in effective viscosity in this layer, and results in the drag reduction. In agreement with this idea, drag reduction of polyelectrolytes increases with increasing concentration of added salt: the salt makes the polymers more flexible, increasing the elastic effects and drag reduction at the same time $[4,11]$. However, the relation between polymer stretching and drag reduction is difficult to study directly, because the polymer stretching is hard to investigate simultaneously in both time and space, and its effect on the effective viscosity is very non-linear [12]. In simulations this is in fact possible, and Benzi et al. have 
shown that indeed the presence of rigid polymers can lead to a linear effective viscosity profile [13].

It is for this reason that we study rigid polymers here: its changes in viscosity are much simpler to understand and describe, since they are only due to the orientation of the polymer molecule. At rest, the orientation of the polymers is random, and the resulting viscosity is high. In a simple shear flow (such as encountered in the viscous sublayer) the polymers orient, which facilitates the flow, and hence the viscosity decreases. Consequently, as a function of shear rate the viscosity decreases: a suspension of rigid polymers is simply shear thinning and shows no elastic effects due to polymer stretching $[4,11,14-18]$. One may anticipate that in a fully turbulent flow the polymers are oriented randomly again, and then the viscosity is high again [8-10].

Turbulent drag reduction by rigid polymers has obtained less attention than flexible polymers [14,15]. Rigid polymers are nonetheless of great interest, since they are inexpensive, environmentally friendly and show significant resistance to mechanical degradation compared to flexible polymers with similar molecular weights. The aim of this work is to investigate whether the linear viscosity mechanism for drag reduction also applies to rod-like polymers, and to see whether this can be related directly to the effect of the orientation of the polymer molecules. We therefore study the turbulent drag-reduction characteristics of the rod-like polysaccharide Xanthan gum [19-21].

\section{Experimental}

\subsection{Materials and experimental setup}

Xanthan gum (XG), purchased from Sigma-Aldrich, Inc., is suspended in tap water. We present here only results for a concentration of $500 \mathrm{ppm}$, as this is close to the optimum concentration; for the concentration dependence, see [8-10]. The rheology of the XG solution is studied on the Rheologica Stress-Tech. The viscosity of XG shows a pronounced shear-thinning behavior (i.e., the viscosity decreases with increasing velocity gradients).

The turbulent drag reduction characteristics of the rod-like polysaccharide XG are investigated using a horizontal closed-loop system [22]. The system consists of two main parts. The first part is made from a stainlesssteel tube equipped with a deferential pressure transducer (DRUCK, PDCR 2111). This pressure transducer is connected to two pressure taps, $6.2 \mathrm{~m}$ apart. The pressure taps are controlled by two sensors arranged along the channel to measure the pressure loss. The measurement range of the pressure sensors is $0-350$ bar. The second part is used to visualize the flow through Particle Imaging Velocimetry (PIV) in a transparent glass tube, around $1.2 \mathrm{~m}$ long with a diameter of $22.5 \mathrm{~mm}$. This tube is made from a borosilicate pipe glass,. The fluid flow is driven by a volumetric pump (PCM, MR13I10) from a reservoir tank of 30 liters. To reduce pressure fluctuations in the closed system, a pressure damper is installed at the outlet of the pump. On the other side, a magnetic flow meter is placed to measure the flow rate. The volumetric pump and valve are used to adjust the flow rate. The pump used here can deliver up to 8 bar (for $0.95 \mathrm{~kW}$ ). The temperature is measured when entering and exiting the system. The temperature is controlled by a heat exchanger and measured by two sensors (ANALOG DEVICES, AD592CN). The velocity profile experiments are performed at $\mathrm{Re}=20000$ and $R e=30000$. For a better quality of results, the velocity profiles of the $\mathrm{XG}$ /water solution are determined in a $22.5 \mathrm{~mm}$ diameter channel at a temperature of $20^{\circ} \mathrm{C}$.

The PIV experiments are performed by placing the glass test tube section in a rectangular plexiglass box to minimize the effect of the refraction of the laser beams. Measurements are taken at a distance of $L_{b}=70 \mathrm{~cm}$ from the glass pipe inlet to the test section. The flow is seeded with the glass particles of $15 \mu \mathrm{m}$ in diameter. An Nd-Yagtype laser is used as a source of illumination and a CCD camera $(1024 \times 1280$ pixel resolution) is used to record flow images. The velocity fields are measured by PIV using DaVis software (from La Vision). The average velocity field parameter is calculated from several instantaneous fields.

\section{Results}

The addition of small amounts of polymer to a turbulent fluid flow causes a large reduction in the frictional drag relative to a pure solvent at the same flow rate. The Fanning friction factor $f$ is defined as

$$
f=\frac{\Delta p \pi^{2} D^{5}}{32 \rho L Q^{2}}
$$

which depends on the density of the solution $\rho$, the bulk velocity $U$ and the wall shear stress $\tau_{w}$. It is experimentally calculated from our measurements of the flow rate $Q$, the pressure drop $\Delta P$ between two locations spaced at a distance of $L$, and inner diameter $D$ of the pipe. Drag reduction (DR) then means that the pressure drop is reduced at the same flow rate or if the flow rate is increased at the same pressure drop. The percentage of DR at a constant flow rate is defined based on the friction factor of the polymer solution and water:

$$
D R \%=\left(\frac{f_{S}-f_{D R}}{f_{S}}\right) \cdot 100,
$$

where $f_{S}$ and $f_{D R}$ are the friction coefficient of the solvent and drag reducing solutions, respectively. We find that the drag reduction due to the addition of $500 \mathrm{ppm}$ XG at $\mathrm{Re}=20000(\mathrm{Re}=30000)$ amounts to $35 \%(40 \%)$.

Drag reduction is accompanied by changes in the velocity profiles. The velocity profiles are usually represented by non-dimensional semilogarithmic coordinates of the fluid velocity $U^{+}$versus distance from the wall $y^{+}$defined as follows:

$$
\begin{aligned}
U^{+} & =\frac{u}{U_{\tau}}, \\
y^{+} & =\frac{y u_{\tau}}{\nu},
\end{aligned}
$$


where $u$ is the local velocity at a distance $y$ from the wall, $\nu$ is the kinematic viscosity of the fluid and

$$
u_{\tau}=\sqrt{\frac{\tau_{w}}{\rho}}
$$

(with $\tau_{w}$ the wall shear stress) designates a wall friction velocity.

Figure 1 shows the measured velocity profiles in the dimensionless coordinates of eqs. (3)-(5) where the solvent viscosity was used to calculate the length scale $y^{+}$. The measured velocity for pure water shows good agreement with the law of the wall $U^{+}=2.5 \ln y^{+}+5.5$. The velocity profiles shows significant changes upon addition of the XG polymer that are in fact similar to those reported for flexible polymers; the profiles go towards Virks' maximum drag reduction velocity profile [23-26]: $U^{+}=11.7 \ln y^{+}-17.0$, that was originally conceived to account for drag reduction of flexible polymers.

The difference between the velocity profiles of the water/XG solution and pure water flows in the logarithmic region increases with DR level and is accompanied by a thickening of the buffer layer. Further away from the wall the velocity of the water/XG solution increases compared to pure water, as is expected since the solution shows drag reduction. The result of the velocity profile measurement clearly demonstrates a thickening of the buffer layer and an increase in the slope of the logarithmic profile in the wall region.

The drag-reduction effect is accompanied by the modification of the turbulent structure. The turbulence intensity of the streamwise and wall-normal velocity fluctuation for water and water/XG solution are presented in fig. 4. Non-dimensionalization of the streamwise and wallnormal velocity fluctuations are made by a friction velocity $\left(u_{\tau}\right)$ :

$$
\begin{aligned}
& u_{r m s}^{\prime}=\frac{\sqrt{\langle u u\rangle}}{u_{\tau}}, \\
& v_{r m s}^{\prime}=\frac{\sqrt{\langle v v\rangle}}{u_{\tau}},
\end{aligned}
$$

where $\langle u u\rangle$ and $\langle v v\rangle$ are turbulence intensities.

Figure 2 shows that the location of the maximum value of streamwise fluctuations for water is very close to the wall and that these velocity fluctuations monotonously decrease from the near-wall to the center of the channel. In drag-reducing flows, the turbulence intensity in the vicinity of the wall is damped in comparison to pure water flow whereas in the buffer layer an increase of the streamwise turbulence intensity occurs. As can be seen in fig. 3, the peak levels of the streamwise velocity fluctuations are shifted away from the wall for drag-reducing solutions and the most characteristic changes occur in the near-wall buffer zone. The normalized turbulence intensity in the streamwise direction in the drag reducing flow shows a shift of the maximum fluctuations towards larger wall distances and the peak itself is broader than for water. On the other hand, the streamwise velocity fluctuations monotonously decrease toward the center of the

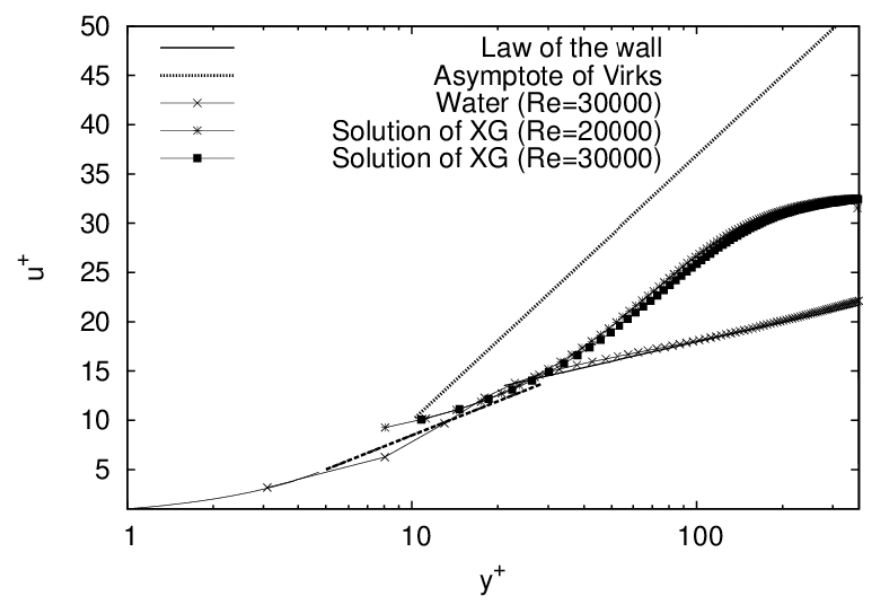

Fig. 1. Average velocity gradients of pure water at $R e=30000$ $(+$ symbols $)$ and water $/ \mathrm{XG}$ solutions at $\mathrm{Re}=20000(\times)$ and $\mathrm{Re}=30000(\mathbf{\square})$ in the usual dimensionless coordinates. The highest line corresponds to Virks' theory, the dotted line to the law of the wall for Newonian fluids.
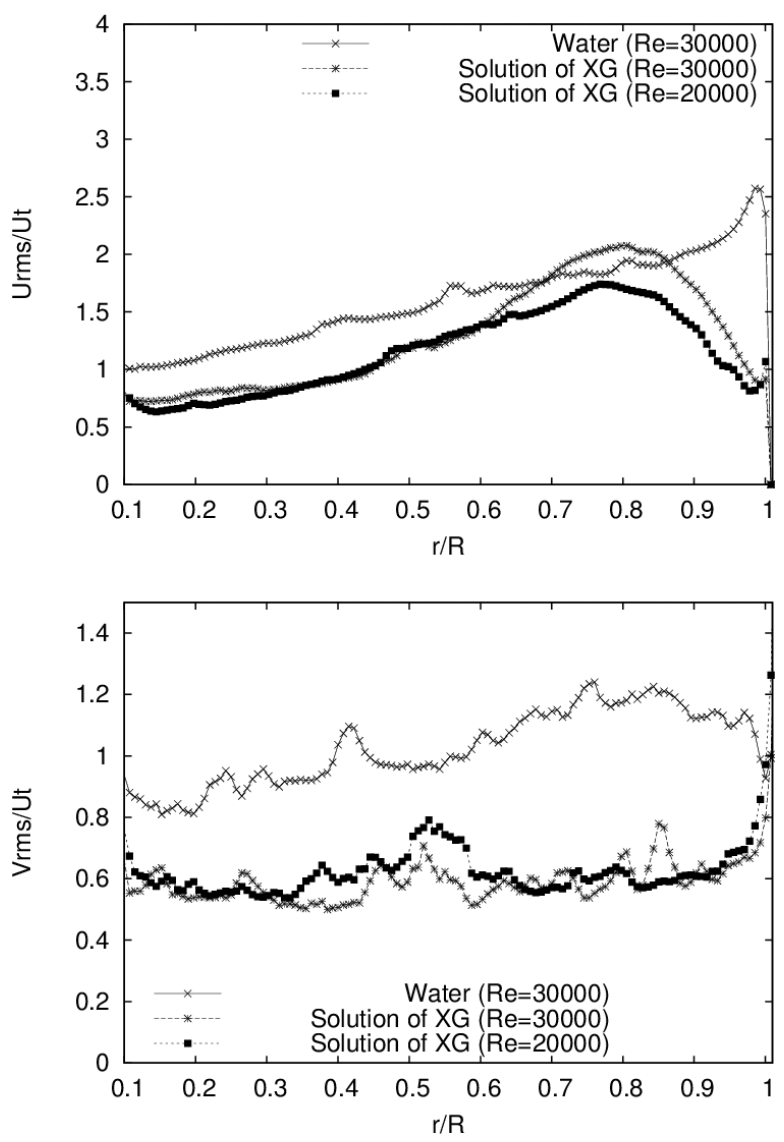

Fig. 2. (Top) Streamwise velocity fluctuations and (bottom) wall-normal velocity fluctuations for water at $R e=30000(\times)$, and water $/ \mathrm{XG}$ solutions at $\operatorname{Re}=20000(\boldsymbol{\square})$ and $\operatorname{Re}=30000(*)$.

channel. This behavior of the streamwise intensity is similar to that found for drag-reducing flexible polymers by Berman [27]. The dimensionless fluctuations in the wallnormal direction are higher for water than for the water/XG solutions. This suggests that the turbulent energy 


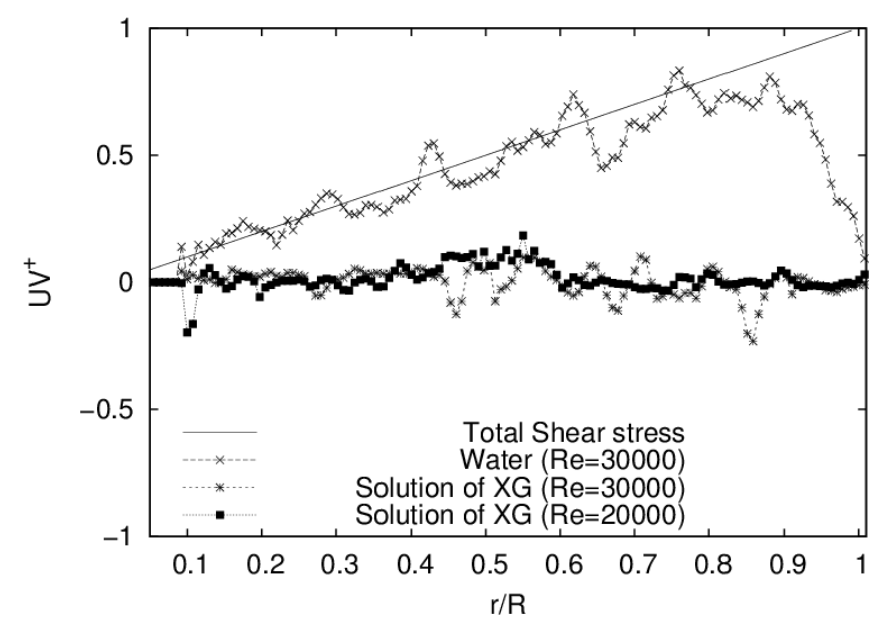

Fig. 3. Profiles of Reynolds shear stress for pure water at $R e=$ $30000(+$ symbols) and water/XG solutions at $\operatorname{Re}=20000(\times)$ and $\operatorname{Re}=30000(\mathbf{\square})$. The solid line corresponds to total shear stress.

transfer perpendicular to the wall direction is decreased by the polymer additive, and that the velocity fluctuations become strongly anisotropic in the drag-reducing flow, the main effect taking place in the buffer layer.

It is widely believed that (flexible) polymer drag reduction is the combined effect of a dampening of the Reynolds stress without generating additional resistance in the viscous sub-layer. Recent studies have confirmed both experimentally and theoretically that Reynolds shear stresses that characterize the turbulent momentum transport are reduced when the drag-reducing polymers are added to the solution. Figure 3 presents the profiles of the Reynolds shear stress $\overline{u^{\prime} v^{\prime}}$ normalized with the wall friction velocity:

$$
U V^{+}=\frac{\overline{u^{\prime} v^{\prime}}}{u_{\tau}^{2}} .
$$

As revealed by the figure, the measured Reynolds stress for water flow is in close agreement with law of the wall. The addition of the XG polymer results in a decrease of the Reynolds stress. As can be seen, the Reynolds stresses are found to be close to zero. This indicates that the momentum transport mechanism of the drag-reducing flow is quite different from that of the Newtonian fluid flow. This is a similar result to the observations of Warholic et al. [28, 29 ], who found the Reynolds stress to be very close to zero for both surfactant and flexible polymer drag-reducing solutions.

The decrease of the Reynolds stress could be explained by a decoupling of the streamwise $\left(u_{r m s}^{\prime}\right)$ and wall-normal $\left(v_{r m s}^{\prime}\right)$ velocity fluctuations. The cross-correlation coefficient for water and water/XG solution is defined as follows:

$$
R_{u v}=\frac{\overline{u^{\prime} v^{\prime}}}{u_{r m s}^{\prime} v_{r m s}^{\prime}},
$$

where $u_{r m s}^{\prime} v_{r m s}^{\prime}$ are the velocity fluctuations in the streamwise and wall-normal directions and $\overline{u^{\prime} v^{\prime}}$ is the Reynolds shear stress coefficient. As can be seen from

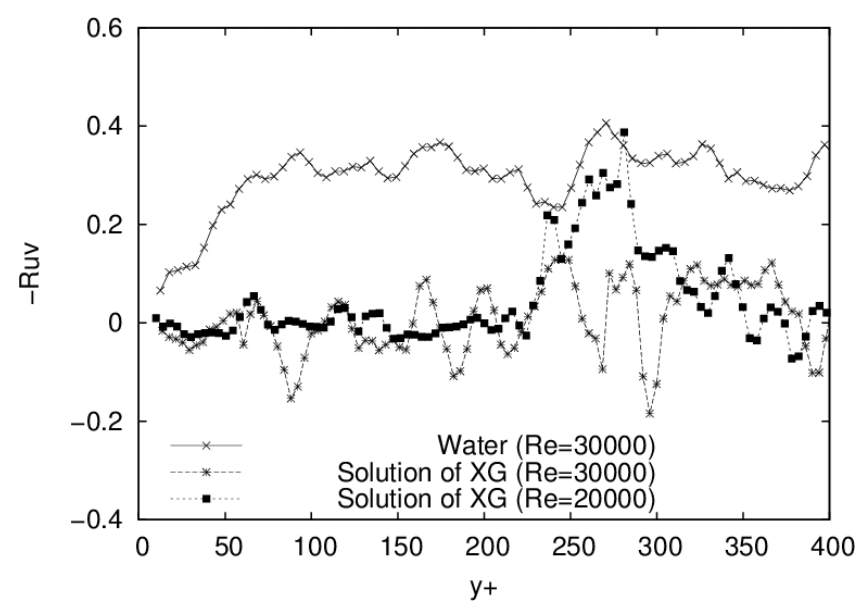

Fig. 4. Cross-correlation coefficients $R_{u v}$ between $u_{r m s}^{\prime}$ and $v_{r m s}^{\prime}$ velocity fluctuations for pure water at $\mathrm{Re}=30000(+$ symbols) and water/XG solutions at $\operatorname{Re}=20000(\times)$ and $\operatorname{Re}=$ 30000

fig. 4, the cross-correlation coefficients between the streamwise and wall-normal fluctuations are drastically reduced with polymers compared to water alone. The loss of correlation between the streamwise and wall-normal velocity components decreases the Reynolds stress, which then leads to drag reduction. Our results for the reduction in Reynolds stress due to a decorrelation between $u_{r m s}^{\prime}$ and $v_{r m s}^{\prime}$ velocity fluctuations are consistent with the descriptions in the literature by Hoyer and Gyr [30], again for flexible polymers.

\subsection{The effective viscosity profile for water/XG}

To understand the nature of the drag-reduction phenomenon, it is important to know the role played by the effective viscosity. In a recent theory of drag reduction in wall turbulence, it was suggested that the presence of polymers leads to an effective viscosity, which increases linearly with the distance from the wall. Such a viscosity profile could decrease the Reynolds stress in the elastic sublayer, resulting in a reduction of the momentum flux to the wall more than it increases the viscous drag, which in turn leads to a net drag reduction. The Reynolds stress deficit occurs in flows of drag-reducing polymer solutions where solvent shear viscosity is employed in the calculation of the distance from the wall $\left(y^{+}\right)$

$$
-\frac{\overline{u^{\prime} v^{\prime}}}{u_{\tau}^{2}}=1-\frac{y}{R}-\frac{\mathrm{d} U^{+}}{\mathrm{d} y^{+}}-G,
$$

where $G$ is the Reynolds stress deficit [31]; here $G$ is the additional stress due to the rigid polymers. Another way of expressing this would be to say that turbulent flow structures are altered so that they can be dominated by viscosity up to higher distances from the wall. The quantity $G$ could then be interpreted by a wall-distance-dependent effective viscosity $\eta$ :

$$
\eta=\frac{\nu_{e f f}}{\nu_{0}}
$$




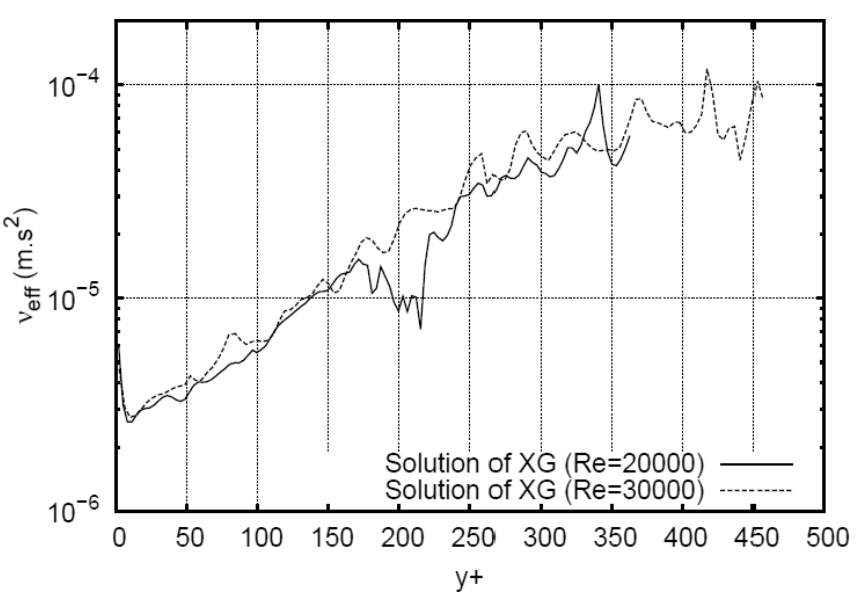

Fig. 5. Effective viscosity of water and water $/ \mathrm{XG}$ at $\mathrm{Re}=$ 30000 .

Earlier, Giesekus [32] suggested that such elastic stresses are attributed to increase the local viscosity, and eq. (8) becomes:

$$
\begin{aligned}
-\frac{\overline{u^{\prime} v^{\prime}}}{u_{\tau}^{2}} & =1-\frac{y}{R}-\left(1+\frac{\nu_{e f f}}{\nu_{0}}\right) \frac{\mathrm{d} U^{+}}{\mathrm{d} y^{+}}-\frac{\overline{u^{\prime} v^{\prime}}}{u_{\tau}^{2}} \\
& =1-\frac{y}{R}-\frac{\nu_{e f f}}{\nu_{0}} \frac{\mathrm{d} U^{+}}{\mathrm{d} y^{+}},
\end{aligned}
$$

where $R$ is the radius of the pipe, $\nu_{e f f}$ is the effective viscosity and $v_{0}$ is the kinematic viscosity of the solvent.

If we consider Reynolds' equation [33] and take into account that the viscosity is variable and that the viscosity fluctuations are low, it becomes

$$
-\frac{\overline{u^{\prime} v^{\prime}}}{u_{\tau}^{2}}=1-\frac{y^{+}}{R^{+}}-\left(1+\frac{\nu_{e f f}}{\nu_{w}}\right) \frac{\mathrm{d} U^{+}}{\mathrm{d} y^{+}},
$$

where $\nu_{w}$ is the wall viscosity. As the results of fig. 3 indicate, $\overline{u^{\prime} v^{\prime}}$ can be neglected and a formula for the spatial variation of the viscosity can be deduced. Figure 5 shows the resulting effective viscosity as a function of the dimensionless wall distance. It reveals that there is an increase in effective viscosity when moving away from the wall when the XG polymer is added. The effective viscosity grows approximately linearly with the wall distance $y^{+}$up to a value of about 30 . Outside the buffer layer, the effective viscosity becomes constant. Based on this experimental result, the drag reduction by rigid polymers can be interpreted as an increase in the local effective viscosity that is small at the wall and increases with increasing distance from the wall.

\section{Conclusions}

In this work, the turbulent drag-reduction characteristics of the rod-like polysaccharide Xanthan gum (XG) were investigated. We performed the drag-reduction measurements using a horizontal closed-loop system. Dragreduction reduction characteristics of $500 \mathrm{ppm}$ water/XG solution were studied at a temperature of $20{ }^{\circ} \mathrm{C}$ at two different Reynolds numbers. The mean velocity profiles were investigated to understand the nature of the dragreduction phenomenon attributed to the XG polymer. For drag-reducing flows, it was found that the addition of XG causes the velocity gradient near the walls to decrease. This decrease also indicates a thickening of the viscous sublayer and gives rise to a lower shear stress or drag when the polymer is added to the flow. Our velocity-profile analysis demonstrates that the interaction between polymer molecules and the turbulent structures takes place in the buffer layer. Further away from the wall the velocity of the water/XG flow increases compared to the Newtonian case, which is consistent with drag reduction. In addition to the velocity-profile measurements, we performed measurements of the turbulence intensities in order to understand the mechanism of the interaction of polymer additives with turbulence. Our results show that the drag reduction leads to a modification of turbulent structures and energy, particularly in the buffer layer. The turbulent kinetic-energy profiles show that the production of kinetic energy is reduced in the drag-reducing water/XG solution flows due to depression of the Reynolds shear stress. In addition, the Reynolds shear stress profiles clearly demonstrate that the Reynolds shear stresses (which characterize the turbulent momentum transport) are drastically reduced by the addition of the drag-reducing XG. The decrease of the Reynolds stress could be explained by a decoupling of the streamwise $u_{r m s}^{\prime}$ and wall-normal $v_{r m s}^{\prime}$ velocity fluctuations. This is suggested to directly lead to a decrease of turbulence production and a reduction of the frictional drag.

Our findings for the XG rigid polymer are very consistent with previous measurements on solutions of flexible polymers, despite the fact that the rheologies of flexible and rigid polymers are very different [16-18]: dilute solutions of flexible polymers show a low shear but a high elongational viscosity, whereas rigid polymers show exactly the opposite: a large shear viscosity (in addition to a strong shear thinning) and a low elongational viscosity [16-18]. Our determination of the effective viscosity profile clearly demonstrates nonetheless that the addition of the rod-like polymer in the Newtonian fluid leads to an increase in the effective viscosity, as for flexible polymers. The increase of this effective viscosity then leads to a similar mechanism for drag reduction for flexible and rigid polymers, as indeed proposed in $[5,11]$. The mechanism itself for the effective viscosity gradient however has to be different between the flexible and rigid polymers. For the former it is believed to be due to polymer stretching. For the latter, it has been speculated [34] that the shear thinning may be at the origin of the effect: in the shear flow of the inner part of the boundary layer, the molecules are aligned and consequently the local, effective viscosity is low, whereas in the bulk of the turbulence the molecules are randomly oriented and consequently the effective viscosity is high. The viscosity profile that we report here is fully consistent with this idea. It is now an interesting question to see whether this also applies to other types of drag reduction, e.g. with surfactants [35] or polyelectrolytes [36]. 


\section{Author contribution statement}

SG and DB designed the research, DK and FH performed the measurements, all authors contributed to the interpretation of the data and the writing of the manuscript.

Open Access This is an open access article distributed under the terms of the Creative Commons Attribution License (http://creativecommons.org/licenses/by/4.0), which permits unrestricted use, distribution, and reproduction in any medium, provided the original work is properly cited.

\section{References}

1. A. Gyr, H.W. Bewersdorff, Drag Reduction of Turbulent Flows by Additives (Kluwer Academic, Dordrecht, 1995).

2. G.C. Liaw, J.L. Zakin, G.K. Patterson, AIChE J. 17, 391 (1971).

3. F. Nieuwstadt, J. Den Toonder, Drag reduction by additives: a review, in Turbulence Structure and Motion, edited by A. Soldati, R. Monti (Springer Verlag, New York, 2001) pp. 269-316.

4. D. Bonn, Y. Amarouchene, C. Wagner, S. Douady, O. Cadot, J. Phys.: Condens. Matter 17, S1195 (2005).

5. M.D. Graham, Phys. Fluids 26, 101301 (2014).

6. J.L. Lumley, Annu. Rev. Fluid. Mech. 1, 367 (1969).

7. J.L. Lumley, J. Polym. Sci.: Macro Rev. 7, 263 (1973).

8. V.S. L'vov, A. Pomyalov, I. Procaccia, V. Tiberkevich, Phys. Rev. Lett. 92, 24 (2004).

9. E. De Angelis, C.M. Casciola, V.S. L'vov, A. Pomyalov, I. Procaccia, V. Tiberkevich, Phys. Rev. E 70, 055301 (2004).

10. R. Benzi, E.S.C. Ching, T.S. Lo, V.S. L'vov, I. Procaccia, Phys. Rev. E 72, 016305 (2005).

11. C. Wagner, Y. Amarouchene, P. Doyle, D. Bonn, EPL 64, $823(2003)$.

12. A. Lindner, J. Vermant, D. Bonn, Physica A: Stat. Mech. Appl. 319, 125 (2003).

13. R. Benzi, E. Ching, E. de Angelis, I. Procaccia, Phys. Rev. E 77, 046309 (2008).

14. M.A. Tracy, R. Pecora, Annu. Rev. Phys. Chem. 43, 525 (1992).
15. J.S. Paschkewitz, C.D. Dimitropoulos, Y.X. Hou, V.S.R. Somandepalli, M.G. Mungal, E.S.G. Shaqfeh, P. Moin, Phys. Fluids 17, 085101 (2005).

16. D. Bonn, J. Meunier, Phys. Rev. Lett. 79, 2662 (1997).

17. S. Rafaï, D. Bonn, A. Boudaoud, J. Fluid Mech. 513, 77 (2004).

18. C. Wagner, Y. Amarouchene, D. Bonn, J. Eggers, Phys. Rev. Lett. 95, 164504 (2005).

19. M.A. Zirnsak, D.V. Boger, J. Non-Newton. Fluid Mech. 79, 105 (1998).

20. N.B. Wyatt, C.M. Gunther, M.W. Liberatire, J. NonNewton. Fluid Mech. 166, 25 (2011).

21. C.H. Hong, H.J. Choi, K. Zhang, F. Renou, M. Grisel, Carbohydr. Polym. 121, 342 (2015).

22. F. Hadri, A. Besq, S. Guillou, R. Makhloufi, J. NonNewton. Fluid Mech. 166, 326 (2011).

23. P.K. Ptasinski, F.T.M. Nieuwstadt, B.H.A.A. Van Den Brule, M.A. Hulsen, Flow Turbul. Combust. 66, 159 (2001).

24. D.T. Walker, W.G. Tiederman, J. Fluid Mech. 218, 377 (1990).

25. J.M.J. Den Toonder, M.A. Hulsen, G.D.C. Kuiken, F.T.M. Nieuwstadt, J. Fluid Mech. 337, 193 (1997).

26. J.L. Zakin, J. Myska, Z. Chara, AIChE J. 42, 3544 (1996).

27. N.S. Berman, Annu. Rev. Fluid Mech. 10, 47 (1978).

28. M.D. Warholic, G.M. Schmidt, T.J. Hanratty, J. Fluid Mech. 388, 1 (1999).

29. M.D. Warholic, H. Massah, T.J. Hanratty, Exp. Fluids 27, 461 (1999).

30. K. Hoyer, A. Gyr, J. Non-Newton. Fluid Mech. 65, 221 (1996).

31. P. Schfimmer, W. Thielen, Chem. Eng. Commun. 4, 593 (1981).

32. H. Giesekus, Structure of turbulence in drag reducing fluids, Lecture Series 1981-86 (Von Kfirmfin Institute for Fluid Dynamics, Rhode-Saint-Gens̃e, Belgium, 1981).

33. M. Lesieur, Turbulence in Fluids (Springer, Dordrecht, 2008).

34. Y. Amarouchene, D. Bonn, H. Kellay, T.S. Lo, V.S. L'vov, I. Procaccia, Phys. Fluids 20, 065108 (2008).

35. J. Drappier, T. Divoux, Y. Amarouchene, F. Bertrand, S. Rodts, O. Cadot, J. Meunier, D. Bonn, Europhys. Lett. 74, 362 (2005).

36. C. Wagner, Y. Amarouchene, P. Doyle, D. Bonn, Europhys. Lett. 64, 823 (2003). 